\title{
A New Simplified Approach for Design of Dual-Band Wilkinson Power Divider with Two and Three Transmission Line Sections Using Only Even-Mode Analysis
}

\author{
Zafar Bedar Khan, Zhao Huiling, Zhang Yimin \\ Dept of Electronics and Information, Northwestern Polytechnical University, Xian, 710072, P.R. China \\ zafarbedarkhan@mail.nwpu.edu.cn, zhhl@nwpu.edu.cn, 18792491553@163.com
}

\begin{abstract}
In this paper, design of dual-band equal-split Wilkinson power divider (WPD) with two and three transmission line (TL) sections is tackled from the perspective of a new simplified mathematical approach as compared to the previously presented work. It is mathematically proven and experimentally verified that only even-mode circuit analysis suffices for outlining the design guidelines for such WPDs which greatly reduces the mathematical complexity. Owing to the cascaded nature of the TL sections and using even-mode analysis, firstly, design equations and design graph are drawn for the two TL sections WPD. The technique is then extended to the design of a three TL sections WPD with simplifications in the circuit, rendering the approach much simpler and mathematically less rigorous (as compared to the previous work). The comparisons of the proposed and previous design approach are drawn. To prove the efficacy of the presented simplified design approach, a two TL sections and a three TL sections WPD are designed at $1 \mathrm{GHz}$ and $2.4 \mathrm{GHz}$ and fabricated. The measured S-parameters show good conformance with the simulated results indicating practical viability of the presented simplified approach. For instance the insertion loss in the two and three TL section is better than 3.2dB. The return loss and isolation between the ports in two TL sections WPD come out to be better than $28 \mathrm{~dB}$ and $29 \mathrm{~dB}$, and in three TL sections is better than $30 \mathrm{~dB}$ and $29 \mathrm{~dB}$ respectively at the two design frequencies.
\end{abstract}

Index Terms - Dual-band, even-mode analysis, transmission line sections, Wilkinson power divider.

\section{INTRODUCTION}

Wilkinson Power divider (WPD) is one of the basic building blocks of most RF front-ends (RFFE) owing to its equal/unequal power division and/or combination capability. With the advent of multiband transceiver systems, research focus has been shifted towards design of multi-band WPDs, digressing from the well-established design guidelines of conventional single frequency band WPD [1]. For example, for dual-band operations (such as, but not limited to, GSM and PCS for cellular phones), many techniques have been proposed for dual-band WPD design encompassing two Transmission lines (TL) sections equal split [2-7], rat-race couplers [8], three TL sections equal-split [9], unequal division [10-11] and meta-material based implementation of WPD [12-13]. Tri and quad- 
band WPDs are proposed in [14-15]. A two TL sections WPD with length equal to one third of the design wavelength was proposed in [2]. The proposed approach, however, was limited to design at the fundamental frequency and its first harmonic only. Addressing this limitation in [3], the author presented closed form equations after extensive mathematics for design of dual-band WPD at any two arbitrary frequencies. A two TL sections equal split WPD, proposed in [3] is shown in Fig. 1(a). Nevertheless in [3] there was a room for improvements in the return loss and port isolation due to poor response. Subsequently, in order to improve S-parameters response like insertion loss, return loss and ports isolation (S21/S31, S11/S22/S33, and S23 respectively), RLC component were used in [47]. Clearly for formulation of generic design guidelines over a wide frequency band, LC components response may vary with the change in frequency and may additionally have increasing parasitic effects as the frequency goes higher. One such attempt avoiding the use of LC components was reported in [9] where a three TL sections equal division WPD operating at two arbitrary frequencies was proposed. The design used only a resistor and a third transmission line section to improve upon the return loss and isolation between the output ports as compared to [3] over a wide range of frequency. This has an advantage in terms of repeatability and easy fabrication when it comes to the micro-strip implementation. However, the method presented in [9] is mathematically extensive and complex owing to formulation of four unknown parameters of the proposed circuit namely $Z_{1}, Z_{2}, Z_{3}$ and $R$ as shown in Fig. 3(a). These unknowns were found out by solving four non-linear transcendental equations derived from the even and odd mode analysis after rigorous mathematical derivations.

In this paper, generic design guidelines for two and three TL sections equal-split WPD for a wide range of frequencies are presented based on a simplified mathematical approach for analysis of the circuit parameters. The analysis throughout is based on the network theory and even-mode analysis only, owing to the cascaded transmission line sections and symmetric nature of equal split WPD structure respectively. Firstly, in order to build up the concept and extend it further, the simpler circuit shown in Fig. 1(a), also presented in [3], is re-analyzed using the network theory (unlike [3]) and the unknown parameters of the circuit are found out through even-mode analysis only.

Generic design graph for a wide range of frequency ratio $\left(m=f_{2} / f_{1}\right.$ where $f_{1} \& f_{2}$ are two arbitrary frequencies) is presented for two TL sections WPD. The idea is then extended to the design of three TL sections WPD as shown in Fig. 3(a) (presented in [9]) with proposed simplifications. With introduction of proposed simplifications in the three TL sections structure of Fig. 3(a) [9], it is shown that the complexity of the analysis for determination of the unknown parameters of the circuit is reduced to half with a fairly comparable operational response of all S-parameters. Experimental verification of the design work based on the proposed simplified design guidelines comes from the results of manufactured dual-band with equal split two and three TL sections WPDs. The operating frequencies are $1 \mathrm{GHz}$ and $2.4 \mathrm{GHz}$ (with frequency ratio $m=f_{2} / f_{l}=2.4$, where $f_{l}$ is assumed to be less than $f_{2}$ ). The measured results show excellent conformance with the simulated results over a wide 
range of the frequency ratio. The trade-offs of the proposed simplifications as compared to [9] are discussed.

\section{PROPOSED ANALYSIS}

\section{A. Two TL Sections WPD}

Equal split two TL sections WPD shown in Fig. 1(a) is re-analyzed to formulate a general guideline for design over a wide range of frequency ratio (m). The main advantage of the presented analysis is that the derived non-linear equations are a function of $m$ (through electrical length $\theta$ which in turn is a function of $m$ ), unlike [3] in which the rigorous mathematical equations are function of the frequency which, if changed, warrants the calculations again. Following mathematical derivations were extended in the next sub-section to a three TL sections WPD design with proposed simplifications which improves the performance of the WPD with two TL sections.

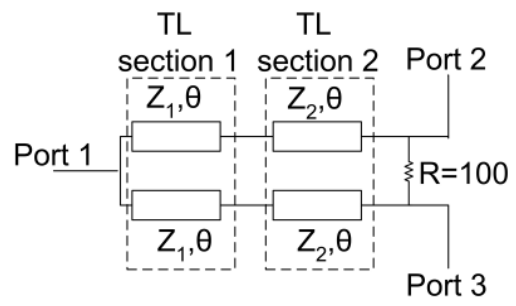

(a)

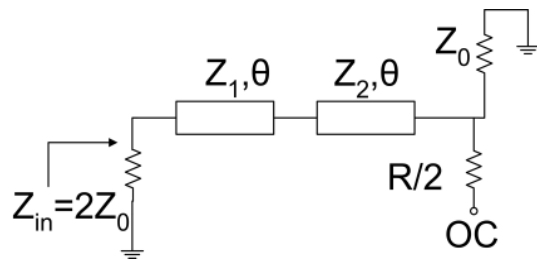

(b)

Fig. 1. WPD with two TL sections (a) structure proposed in [3] and (b) bisected (half) circuit for even mode analysis.

\section{i. Even Mode Analysis For Dual Band Operation}

Due to symmetry of the circuit shown in Fig. 1(a), it can be bisected with input impedance equal to $2 Z_{0}$ for even mode analysis as shown in Fig. 1(b). Treating the circuit in Fig. 1(b) as a cascaded network of two TLs with unknown impedance of $Z_{1}$ and $Z_{2}$ (normalized impedances of $z_{1}$ and $z_{2}$ respectively), the $\mathrm{ABCD}$ parameters can be given as follows

$$
\left(\begin{array}{ll}
\mathrm{A} & \mathrm{B} \\
\mathrm{C} & \mathrm{D}
\end{array}\right)=\left(\begin{array}{cc}
\cos \theta & \mathrm{j \textrm {z } _ { 1 } \operatorname { s i n } \theta} \\
\frac{\mathrm{j} \sin \theta}{\mathrm{z}_{1}} & \cos \theta
\end{array}\right)\left(\begin{array}{cc}
\cos \theta & \mathrm{j \textrm {z } _ { 2 }} \sin \theta \\
\frac{\mathrm{j} \sin \theta}{\mathrm{z}_{2}} & \cos \theta
\end{array}\right)
$$

So that

$$
\begin{aligned}
& \mathrm{A}=\cos ^{2} \theta-\frac{\mathrm{z}_{1} \sin ^{2} \theta}{\mathrm{z}_{2}} \\
& \mathrm{~B}=\mathrm{j} \cos \theta \sin \theta\left(\mathrm{z}_{1}+\mathrm{z}_{2}\right) \\
& \mathrm{C}=\mathrm{j} \cos \theta \sin \theta\left(\frac{1}{\mathrm{z}_{1}}+\frac{1}{\mathrm{z}_{2}}\right) \\
& \mathrm{D}=\cos ^{2} \theta-\frac{\mathrm{z}_{2} \sin ^{2} \theta}{\mathrm{z}_{1}}
\end{aligned}
$$

In order to ensure effective operation of the WPD at two arbitrary frequencies of $f_{1}$ and $f_{2}=m f_{1}$ (where $m$ is the frequency ratio), the input impedance must be matched to the output impedance. As per Fig. 1(b) the input impedance can be written as 


$$
\mathrm{Z}_{\text {in }}=\frac{\mathrm{B}+\mathrm{AZ}_{0}}{\mathrm{D}+\mathrm{CZ} \mathrm{Z}_{0}}=2 \mathrm{Z}_{0}
$$

Putting values of the A, B, C and D from (2)-(5) in (6), simplifying and separating the real and imaginary parts, following two non-linear equations can be derived:

$$
\begin{aligned}
& \mathrm{z}_{1} \mathrm{z}_{2}+\left(\mathrm{z}_{1}^{2}-2 \mathrm{z}_{2}^{2}\right) \tan ^{2} \theta=0 \\
& 2\left(\mathrm{z}_{1}+\mathrm{z}_{2}\right)-\mathrm{z}_{1} \mathrm{z}_{2}\left(\mathrm{z}_{1}+\mathrm{z}_{2}\right)=0
\end{aligned}
$$

Here we have two nonlinear equations with two unknowns. No further analysis is required indicating that even-mode analysis is enough which reduces the mathematical complexity. It is worth mentioning here the very fact that taking equal division, only resistor as isolation element and system characteristic impedance of $Z_{0}=50 \Omega$ into consideration, makes it possible to select $R=100 \Omega$ based on extensive analysis [1,4-7] for effective matching and sufficient isolation between the input and output ports thus avoiding the odd-mode analysis. However for LC as isolation elements [4-5, 7] and/or unequal division [9, 10] and/or more than one resistor as isolation element [11-12], as design specifications, odd-mode analysis cannot be avoided.

Equations (7)-(8) can be simultaneously solved for different electrical lengths $(\theta)$. Note that the electric length $\theta$ can be converted to physical length $l$ by the relation $\theta=\beta l$, where $\beta$ is the wave number. The physical length $(l)$ formula is given in (9) [3] and at the lower design frequency $\left(f_{l}\right)$ the electrical length $(\theta)$ which satisfies (7) and (8) is obtained from (10).

$$
\begin{gathered}
I_{f 1}=I_{f 2}=\frac{\mathrm{n} \pi}{\beta_{1}+\beta_{2}} \\
\theta_{\mathrm{f} 1}=\frac{\mathrm{n} \pi}{1+\mathrm{m}}
\end{gathered}
$$

Where $n$ is a constant and its value is kept at 1 for smaller size, and $m$ is the frequency ratio. The physical lengths of the TL sections are kept equal [2-3].

Since electrical length $(\theta)$ is now known, the two non-linear equations (7) and (8) are simultaneously solved for the unknowns $z_{1}$ and $z_{2}$. Fig. 2 presents a design graph in which the normalized impedances $z_{1}$ and $z_{2}$ are plotted against a wide range of frequency ratio $\left(m=f_{2} / f_{1}\right)$ on the $\mathrm{x}$ axis.

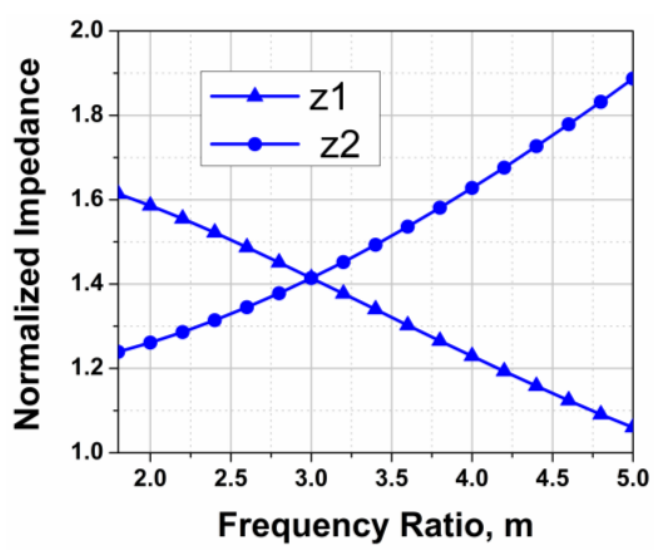

Fig. 2. Normalized impedances vs. frequency ratio $(m)$ formulated through proposed method in sub section 2.1 . 
Contrary to [3], Fig. 2 shows a design graph for two TL section equal split WPD over a wide range of frequency ratio $(\mathrm{m})$ from 1.8 to 5 . It is evident here that it is very easy to design and manufacture a two TL sections equal split WPD with the proposed general design guideline.

\section{B. $\quad$ Three TL Sections WPD}

The complex and mathematically rigorous approach presented in [9] for determination of the unknown parameters of the circuit shown in Fig. 3(a) can be greatly simplified.

Here, the proposed methodology in the previous sub-section is extended to the design of three TL sections WPD with proposed simplifications in the circuit shown in Fig. 3(a).

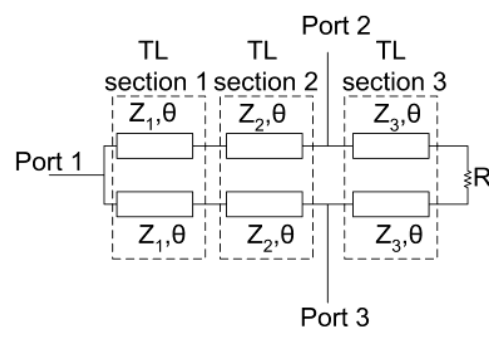

(a)

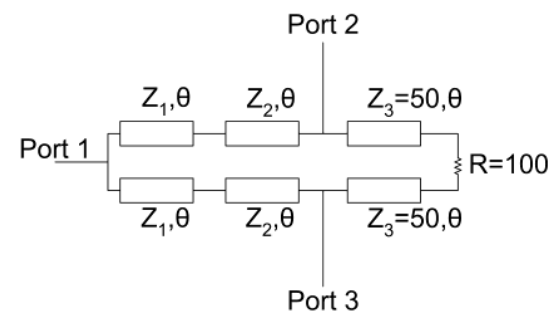

(b)

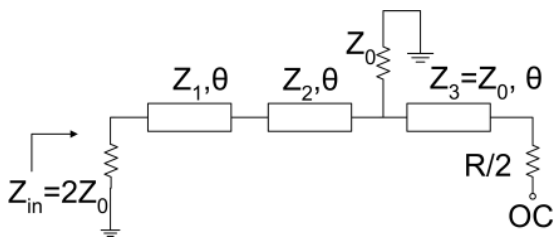

(c)

Fig. 3. WPD comprising of three section TL structure (a) Structure in [9] (b) Structure with proposed simplifications and (c) Bisected circuit for even mode analysis.

\section{i. Proposed Simplification}

The complexity of analysis in Fig. 3(a) owes itself to the variable impedances of the additional TL section 3 and isolation resistor. It is proposed and proven here that for all practical purposes, the third TL section in such kind of three TL sections equal split WPD design as shown in Fig. 3(a), can always be treated as a $50 \Omega$ extension TL to increase the isolation between the output ports (ports $2 \& 3$ ) and the return loss. In addition, for similar reasoning presented in section IIA, since the design under consideration has equal-split power ratio and system characteristic impedance of $Z_{0}=50 \Omega$, no LC components and only one isolation resistor $(R)$, the value of $R$ can be fixed to the conventional value of $100 \Omega$ [1,4-7] without compromising the performance of the dual-band WPD. With the proposed simplifications as shown in Fig. 3(b), the number of unknowns in the circuit is reduced to two, namely $Z_{1}$ and $Z_{2}$, as compared to four in Fig. 3(a). It implies that the complexity can be brought down to half as compared to [9] since the number of non-linear equations to be solved now can be reduced to two instead of four. The two equations can be obtained by even mode analysis only, based on half circuit in Fig. 3(c).

ii. Even mode analysis for dual-band operation

There is no current flow in the symmetric plane of the circuit shown in Fig. 3(b) due to application of two signals of same amplitude and phase at the two output ports. So owing to symmetry, the circuit 
was bisected at the middle as shown in Fig. 3(c).

With input impedance as $2 Z_{0}$ at port 1 and treating the circuit in Fig. 3(c) as cascaded network of two TLs and a third shunt TL with impedances of $Z_{1}, Z_{2}$ and 50 (normalized impedances of $z_{1}, z_{2}$ and 1) respectively, the $\mathrm{ABCD}$ parameters can be derived. Mathematically,

$$
\left(\begin{array}{ll}
A & B \\
C & D
\end{array}\right)=\left(\begin{array}{cc}
\cos \theta & \mathrm{jz} \sin \theta \\
\frac{\mathrm{j} \sin \theta}{\mathrm{z}_{1}} & \cos \theta
\end{array}\right)\left(\begin{array}{cc}
\cos \theta & \mathrm{jz} \mathrm{z}_{2} \sin \theta \\
\frac{\mathrm{j} \sin \theta}{\mathrm{z}_{2}} & \cos \theta
\end{array}\right)\left(\begin{array}{cc}
1 & 0 \\
\mathrm{j} \tan \theta & 1
\end{array}\right)
$$

So that

$$
\begin{aligned}
& \mathrm{A}=\cos ^{2} \theta-\frac{\mathrm{z}_{1} \sin ^{2} \theta}{\mathrm{z}_{2}}-\left(\mathrm{z}_{1}+\mathrm{z}_{2}\right) \sin ^{2} \theta \\
& \mathrm{B}=\mathrm{j}\left(\mathrm{z}_{1}+\mathrm{z}_{2}\right) \cos \theta \sin \theta \\
& \mathrm{C}=\mathrm{j}\left(\frac{1}{\mathrm{z}_{1}}+\frac{1}{\mathrm{z}_{2}}+1\right) \cos \theta \sin \theta-\frac{\mathrm{j} \mathrm{z}_{2} \sin ^{2} \theta \tan \theta}{\mathrm{z}_{1}} \\
& \mathrm{D}=\cos ^{2} \theta-\frac{\mathrm{z}_{2} \sin ^{2} \theta}{\mathrm{z}_{1}}
\end{aligned}
$$

The input impedance of the circuit in Fig. 3(c) can be expressed as

$$
\mathrm{Z}_{\text {in }}=\frac{\mathrm{B}+\mathrm{AZ_{0 }}}{\mathrm{D}+\mathrm{CZ} \mathrm{Z}_{0}}=2 \mathrm{Z}_{0}
$$

Putting values of the A, B, C and D from (12)-(15) in (16) simplifying and separating the real and imaginary parts, following two non-linear equations can be derived:

$$
\begin{aligned}
& \left(2 z_{2}^{2}-z_{1}^{2}-z_{1}^{2} z_{2}-z_{1} z_{2}^{2}\right) \tan ^{2} \theta-z_{1} z_{2}=0 \\
& 2\left(z_{2}+z_{1}+z_{1} z_{2}-z_{2}^{2} \tan ^{2} \theta\right)-z_{1}^{2} z_{2}-z_{1} z_{2}^{2}=0
\end{aligned}
$$

Similar to section IIA, at this point there are two equations with two unknowns. The analysis is complete and no further computation is required for formulation of unknown parameters thus circumventing the odd-mode analysis. Contrary to [9], the mathematical complexity has been greatly reduced. Equations (17) and (18) are solved simultaneously with electrical length $(\theta)$ given in (10) to obtain the unknowns $z_{1}$ and $z_{2}$.

Comparison of the normalized impedances varying with wide range of frequency ratio $\left(m=f_{2} / f_{l}\right)$ calculated through the proposed simplified method and [9] is illustrated in Fig. 4. It is evident here that by the introduction of the proposed simplifications, the difference between the unknown parameters is not much as compared to [9]. For example, it is clearly seen in Fig. 4 that the maximum difference between the normalized impedance values of $z_{1}$ and $z_{2}$ is less than $9 \%$ and $3 \%$ respectively. Whereas for $m=2.3$ to $m=5$, the maximum difference between the normalized impedance values of $z_{3}$ and $r$ is less than $15 \%$ and $20 \%$ respectively. The proposed design guidelines are also valid for a wide range of frequency ratio $(m)$ i.e. from 2 to 5 . An additional advantage is that it replaces the resistor with non- standard values in [9] with a conventional and standard value of $100 \Omega$ which is easy to find in different packages. 


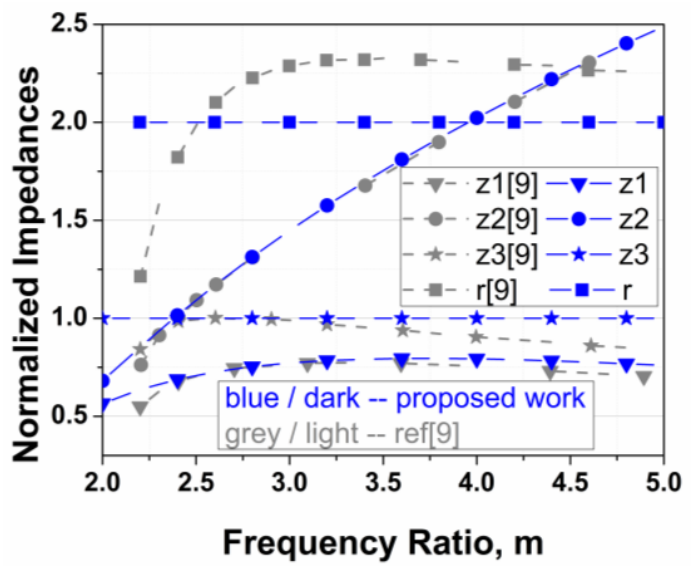

Fig. 4. Comparison of normalized impedances vs. frequency ratio $(m)$ formulated through proposed work and [9].

\section{C. $\quad$ Comparison of Proposed Technique with Referenced Work}

Table I gives the comparison of the parameters calculated through the proposed method (Fig. 2 \& 4) with other referenced works having similar equal split WPD structure with two and three TL sections. It is clear from the Table I that following the proposed simplified technique presented in section IIA \& $\mathrm{B}$, the difference between the calculated unknown parameters is very less thus indicating the usefulness of the proposed analysis.

TABLE I: COMPARISON OF THE CIRCUIT PARAMETER VALUES OBTAINED THROUGH ANALYSIS OF SIMILAR TWO AND THREE TL SECTIONS EQUAL SPLIT WPD PRESENTED IN DIFFERENT REFERENCES WITH THE PROPOSED TECHNIQUE

\begin{tabular}{|c|c|c|c|c|c|c|}
\hline \multirow{2}{*}{ Work } & \multirow{2}{*}{$\begin{array}{l}\text { Isolation } \\
\text { Elements }\end{array}$} & \multirow{2}{*}{$\begin{array}{l}\text { Frequency Ratio } \\
\qquad(m=\mathbf{f} 2 / \mathbf{f} 1)\end{array}$} & \multicolumn{4}{|c|}{ Parameters } \\
\hline & & & $\mathrm{Z}_{1}(\Omega)$ & $\mathrm{Z}_{2}(\Omega)$ & $\mathrm{Z}_{3}(\Omega)$ & $\mathrm{R}(\Omega)$ \\
\hline $\operatorname{Ref}[2,3], 2$ TL Sections & $\mathrm{R}$ & \multirow{2}{*}{2} & 79.37 & 63.00 & \multirow{2}{*}{ N/A } & 100 \\
\hline Proposed Technique (Fig. 2) & $\mathrm{R}$ & & 79.29 & 63.06 & & 100 \\
\hline $\operatorname{Ref}[4,7], 2$ TL Sections & RLC & \multirow{2}{*}{1.8} & 80.70 & 62.00 & \multirow{2}{*}{ N/A } & 100 \\
\hline Proposed Technique (Fig. 2) & $\mathrm{R}$ & & 80.72 & 61.94 & & 100 \\
\hline Ref [9] , 3 TL Sections & $\mathrm{R}$ & \multirow{2}{*}{2.6} & 36.45 & 58.60 & 50.3 & 104.7 \\
\hline Proposed Technique (Fig. 4) & $\mathrm{R}$ & & 36.48 & 58.62 & 50 & 100 \\
\hline
\end{tabular}

\section{EXPERIMENTAL DEMONSTRATION OF THE PROPOSED TECHNIQUE}

The proposed design guidelines in sections IIA \& B for two and three TL sections equal split WPD respectively are shown to be conveniently utilized for design of both types of dual-band equal split WPD with $m=2.4$, operating at $f_{l}=1 \mathrm{GHz}$ and $f_{2}=2.4 \mathrm{GHz}$ (note that $m=3$, is a special case where the WPD designed through proposed technique can be simply replaced by a conventional $\lambda / 4$ transformer which has a property of operating at frequency $f_{0}$ and its first odd harmonics $3^{*} f_{0}$ [4]). Especially in the design of three TL sections WPD, it is practically shown that there is no compromise on the operational parameters (e.g. isolation between port $2 \& 3$ and return loss) while using the proposed technique as compared to [9]. Based on design graphs of Fig. 2 and Fig. 4, the values of the circuit parameters for two and three TL sections WPD respectively are summarized in Table II. 
TABLE II: SUMMARY OF THE IMPEDANCE VALUES (AFTER MULTIPLYING WITH CHARACTERISTIC IMPEDANCE OF 50 2 ) FOR TWO AND THREE TL SECTIONS WPD

\begin{tabular}{|c|c|c|}
\cline { 2 - 3 } \multicolumn{1}{c|}{} & \multicolumn{2}{c|}{$\boldsymbol{m = 2 . 4}\left(f_{1}=1 \mathrm{GHz}\right.$ and $\left.f_{2}=2.4 \mathrm{GHz}\right)$} \\
\hline & Two TL sections & Three TL sections \\
\hline$Z_{l}(\Omega)$ & 76.10 & $\begin{array}{c}\text { Ref: Fig. 4 } \\
\text { (proposed work })\end{array}$ \\
\hline$Z_{2}(\Omega)$ & 65.73 & 34.40 \\
\hline$Z_{3}(\Omega)$ & N/A & 50.76 \\
\hline$R(\Omega)$ & 100 & 50 \\
\hline
\end{tabular}

The electrical length is, $\theta=53^{0}$ when $m=2.4$. ADS software was used for all simulation and layout work. Subsequently, the two circuits were fabricated on a substrate with dielectric constant of 2.2 and thickness of $0.8 \mathrm{~mm}$. The fabrication of the PCBs was carried out on a Milling PCB prototype machine with precision, repeatability tolerance and mechanical resolution of $\pm 20 \mu \mathrm{m}, \pm 5 \mu \mathrm{m}$ and $\pm 0.8 \mu \mathrm{m}$ respectively. The TL widths and lengths corresponding to the impedances mentioned in Table II were calculated accordingly. $Z_{0}$ is taken to be $50 \Omega$ as mentioned earlier.

Fig. 5 shows the photographs of the fabricated dual-band equal split WPD with two and three TL sections. As stated earlier, the physical lengths of the TL sections were kept equal [3]. The size of the designed two TL sections WPD is $7 \mathrm{~cm} \times 4.5 \mathrm{~cm}$ which is comparable to the two examples presented in [4] whose sizes are $6.4 \mathrm{~cm} \times 3.4 \mathrm{~cm}$ and $7.3 \mathrm{~cm} \times 3.8 \mathrm{~cm}$ respectively. The exact size of three TL section WPD in [9] is not given however the designed three TL sections WPD is $10 \mathrm{~cm} \times 3.8 \mathrm{~cm}$ in dimensions.

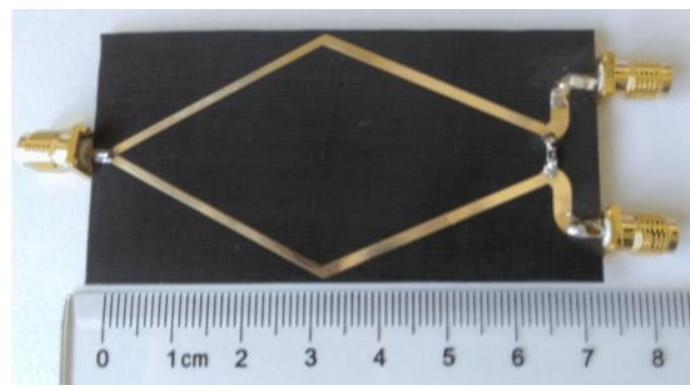

(a)

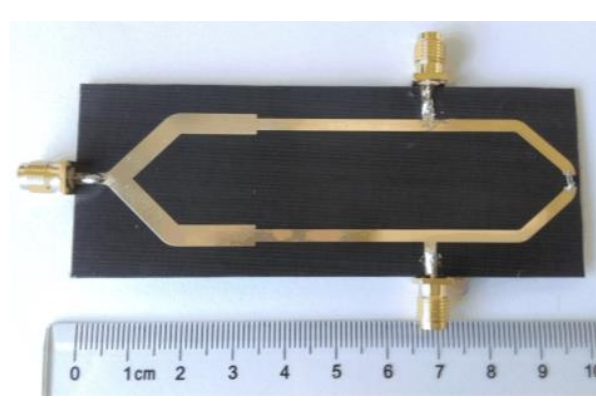

(b)

Fig. 5. Fabricated dual-band equal split WPD operating at frequencies of $1 \mathrm{GHz}$ and $2.4 \mathrm{GHz}$ (frequency ratio $m=2.4$ ) (a) with two TL sections and (b) with three TL sections.

\section{MEASUREMENT RESULTS AND DISCUSSIONS}

The measurements were performed on Agilent's Phase Network Analyzer (PNA) model no. E8363B.

\section{A. Two TL Sections WPD}

A good agreement between the simulated and measured results can be noticed in Fig. 6 (a-e) confirming the authenticity of the design guidelines given in Fig. 2 for two TL sections WPD. From Fig. 6(a) it can be seen that magnitude of return loss at port 1 (S11) is around $30 \mathrm{~dB}$ and $23 \mathrm{~dB}$ at $1 \mathrm{GHz}$ 


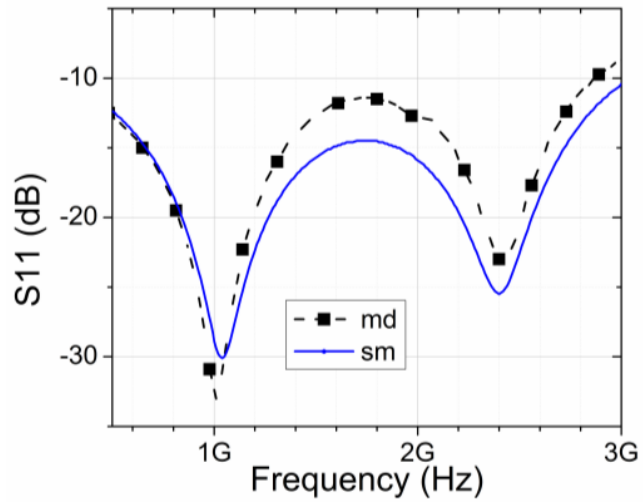

(a)

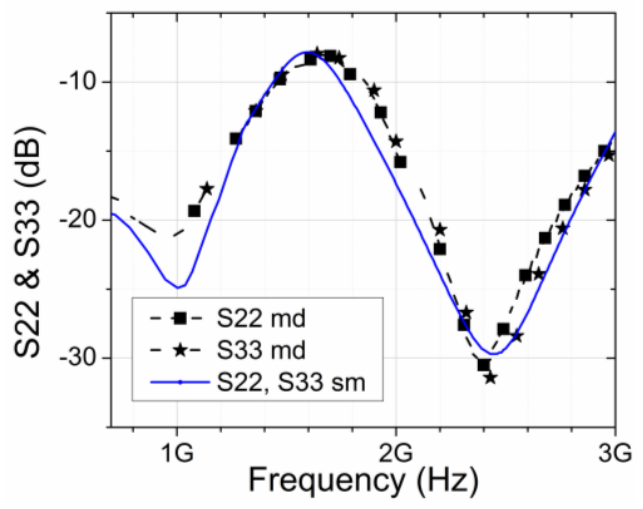

(c)

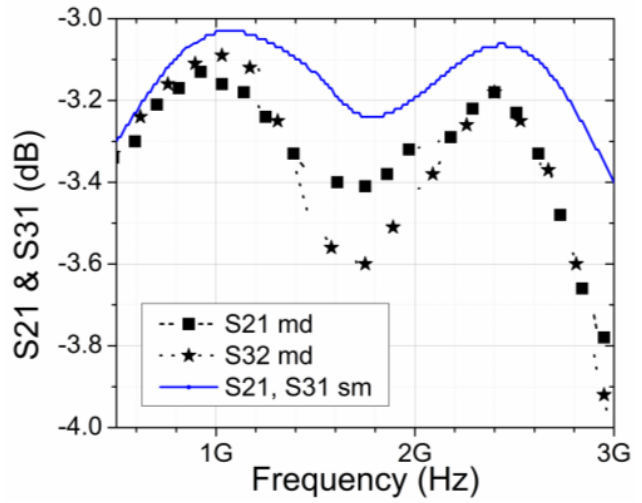

(b)

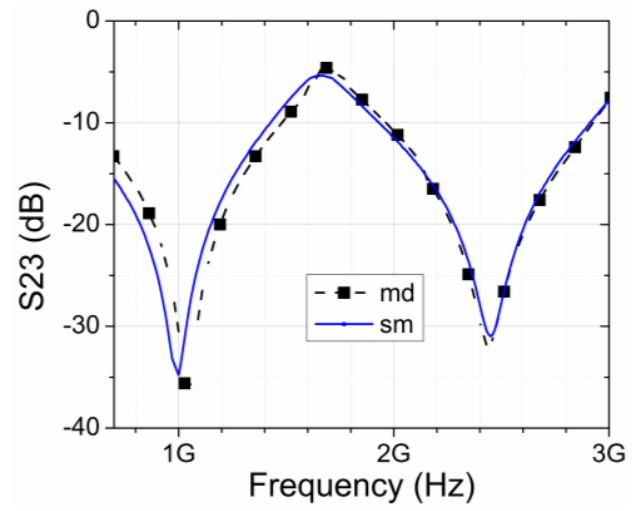

(d)

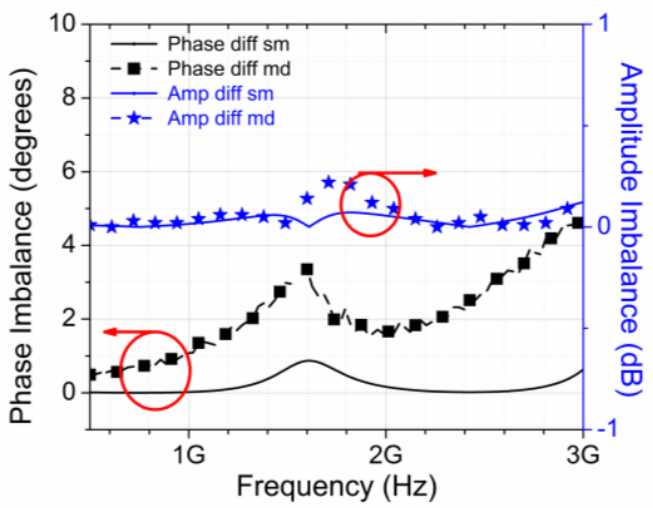

(e)

Fig. 6. Comparison of simulated and measured response of two TL sections WPD. $(\mathrm{md}=$ measured, $\mathrm{sm}=$ simulated) Frequency response of (a) return loss at port 1 (S11) (b) insertion losses (S21 \& S31) (c) return losses (S22 \& S33) (d) output port isolation (S23) and (e) Phase imbalance $(<$ S21 - < S31)and Amplitude imbalance $(|\mathrm{S} 21|-|\mathrm{S} 31|)$ at the two output ports.

and $2.4 \mathrm{GHz}$ respectively showing good impedance matching at port 1 with a maximum error of less than $9 \%$ between the simulated and measured results.

Symmetry of the presented two TL section WPD can be inferred from almost overlapping measured responses of S21 \& S31 (with mutual difference of less than 2\% as in Fig. 6(b)) and S22 \& S33 (Fig. 6(c)) at the design frequencies. Output port isolation is yet another important parameter which is shown in Fig. 6(d). Good isolation of $\mathrm{S} 23=35 \mathrm{~dB}$ and $30 \mathrm{~dB}$ is observed at both operating frequencies respectively. Fig. 6(e) shows the phase and amplitude imbalance of the output signal at port 2 and 3. 
As expected the amplitude difference between the transmitted signal at port 2 and 3 (|S21|-|S31|) is close to zero at the two design frequencies. Whereas the phase difference is about $1.5^{0}$ and $2^{0}$ at 1 $\mathrm{GHz}$ and $2.4 \mathrm{GHz}$ respectively, being reasonably acceptable.

\section{B. $\quad$ Three TL Sections WPD}

Validity of the simplified design guidelines of Fig. 4 is established through conformance of the simulated and measured S-parameters response presented in Fig. 7(a-e).

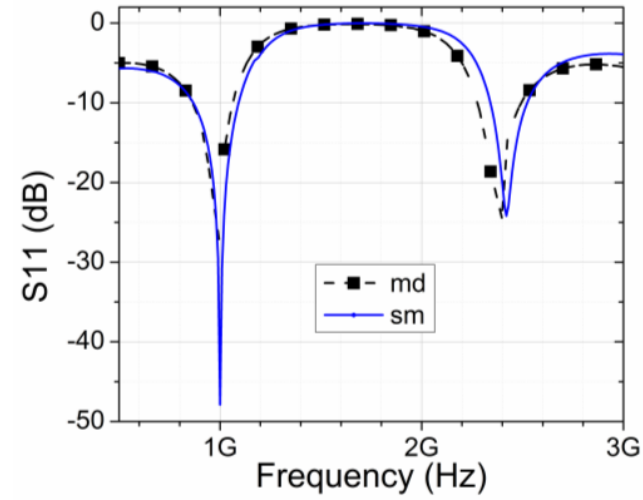

(a)

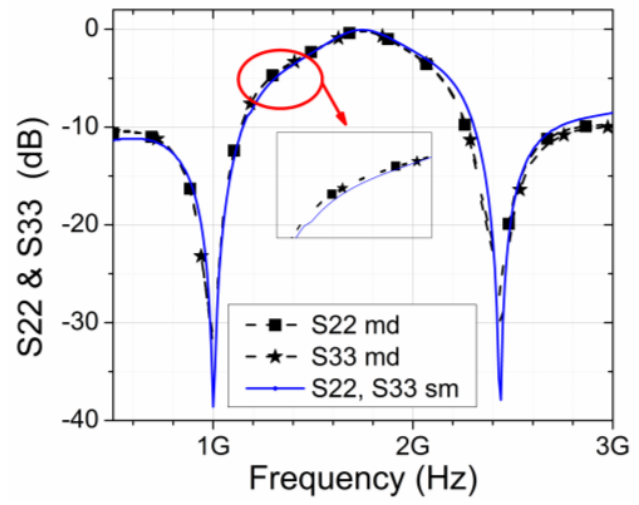

(c)

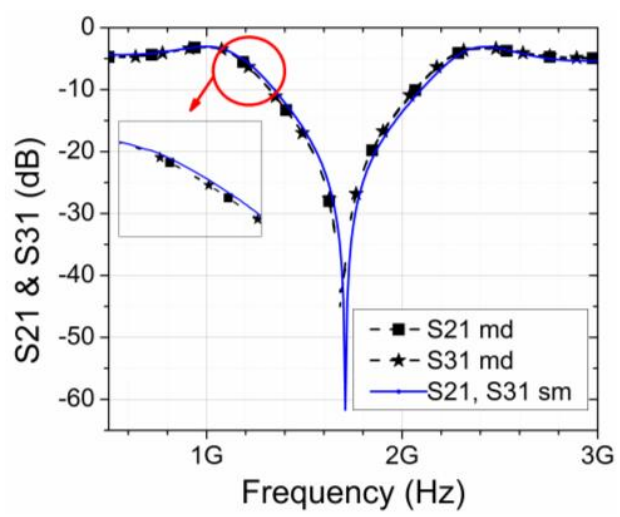

(b)

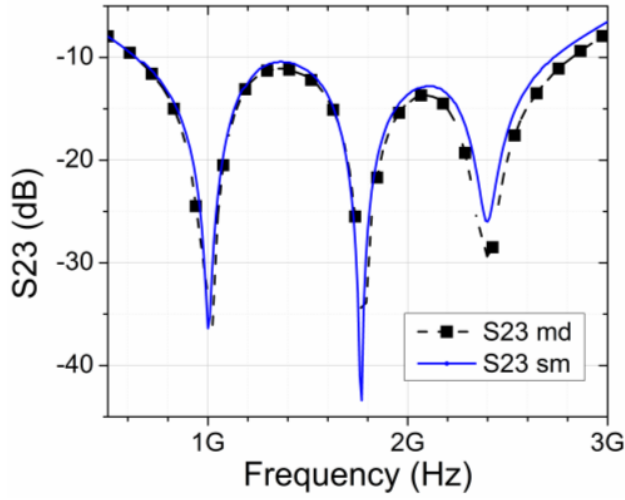

(d)

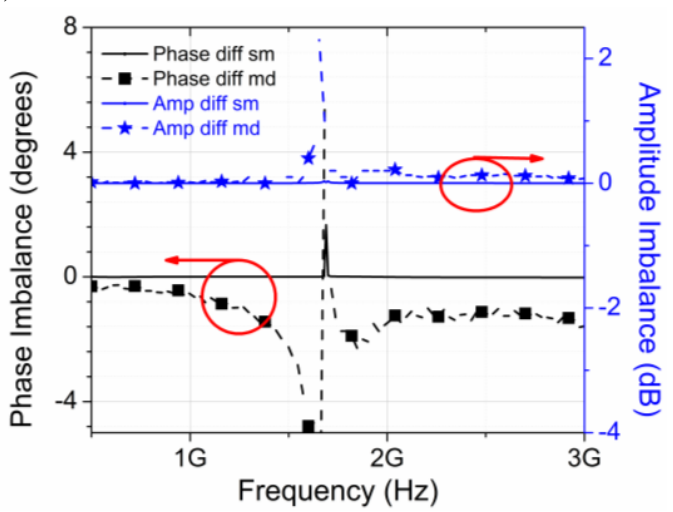

(e)

Fig. 7. Comparison of simulated and measured response of three TL section WPD. ( $\mathrm{md}=$ measured, sm $=$ simulated) Frequency response of (a) return loss at port 1 (S11) (b) insertion losses (S21 \& S31) (c) return losses (S22 \& S33) (d) output port isolation (S23) and (e) Phase imbalance $(<$ S21- < S31) and Amplitude imbalance $(|\mathrm{S} 21|-|\mathrm{S} 31|)$ at the two output ports.

Good impedance match at port 1 is shown in Fig. 7(a) where the $\mathrm{S} 11=31 \mathrm{~dB}$ at $1 \mathrm{GHz}$ (although the simulated $\mathrm{S} 11=48 \mathrm{~dB}$, but measured one is good enough) and $\mathrm{S} 11=24 \mathrm{~dB}$ at $2.4 \mathrm{GHz}$. Almost equal 
division of signal is depicted in Fig. 7(b) with insertion loss S21 and S31 equal to $3.06 \mathrm{~dB}$ and 3.12 $\mathrm{dB}$ at the two design frequencies respectively. Return loss at port $2 \& 3$ (S22 and S33) is shown to be $30 \mathrm{~dB}$ at both design frequencies in Fig. 7(c). Here also, the symmetry of the three TL sections WPD circuit is evident from Fig. 7(b \& c) due to the overlapping response of the insertion loss and return loss at output port $2 \& 3$ respectively (shown in the inset graphs). Fig. 7(d) depicts the output port isolation (S23), which comes out to be $36 \mathrm{~dB}$ and $29 \mathrm{~dB}$ at $1 \mathrm{GHz}$ and $2.4 \mathrm{GHz}$ respectively closely following the simulated results. The proposed WPD has good response for a fractional BW of $20 \%$ and $8 \%$ at $1 \mathrm{GHz}$ and $2.4 \mathrm{GHz}$ respectively. Last but not the least Fig. $7(\mathrm{e})$ shows the phase and amplitude imbalance of the output signal at port 2 and 3 for 3TL sections WPD. The measured phase and amplitude imbalance is $-0.65^{\circ}, 0 \mathrm{~dB}$ and $-1.2^{0}, 0.1 \mathrm{~dB}$ at $1 \mathrm{GHz}$ and $2.4 \mathrm{GHz}$ respectively.

From the comparison of Fig. $6 \&$ 7, it can be drawn that in general the S-parameter response of the dual-band WPD is better in the three TL sections design as claimed in [9]. Although two TL sections design works well at the design frequencies, S-parameters response, especially the output port isolation (S23) is improved in the three TL sections design.

\section{CONCLUSION}

Generalized design guidelines are presented for two and three TL sections equal split WPDs based on a new simplified, mathematically much less rigorous approach which only requires even-mode circuit analysis for evaluation of unknown impedances. For two TL sections WPD, treating the circuit as cascaded network the unknown impedances are found out by even-mode analysis. The mathematical approach is different than [3] through which, for equal split ratio, the values of impedances of the TLs are formulated over a wide range of frequency ratio. The established technique is conveniently extended to three TL sections WPD design where simplifications were introduced in the circuit presented in [9], greatly reducing the mathematical complexity (to half) as compared to [9] without compromising the performance of the WPD. It was established mathematically and was proven experimentally that in such kind of WPD, the third section of the TL can always be replaced with $50 \Omega$ impedance and the isolation resistor's value can be $100 \Omega$ as in conventional WPD design. With these simplifications, even mode analysis sufficed to find out the unknown parameters. Here too, the design guidelines covered a wide range of frequency ratio. Dual-band equal spilt WPDs with two and three TL sections operating at $1 \mathrm{GHz}$ and $2.4 \mathrm{GHz}$ were designed and fabricated based on the presented guidelines respectively. The measured response showed very good conformance with the simulated results with excellent performance parameters. The presented approach can serve as generic, much simpler guidelines for design of such dual-band equal split two and three TL sections WPDs.

\section{REFERENCES}

[1] Pozar, D. M., Microwave Engineering, second edition, Wiley, New York, 1998.

[2] Chow, Y. L. and Wan, K. L., "A transformer of one-third wavelength in two sections-for a frequency and its first harmonic," IEEE Microw. Wireless Compon. Lett., Vol. 12, No. 1, 22-23, Jan. 2002.

[3] Monzon, C., "A small dual-frequency transformer in two sections," IEEE Trans. Microw. Theory Tech., Vol. 51, No. 4, 1157-1161, Apr. 2003. 
[4] Wu, L., Sun, Z., Yilmaz, H., and Berroth, M., “A dual-frequency Wilkinson power divider," IEEE Trans. Microw. Theory Tech., Vol. 54, No. 1, 278-284, Jan. 2006.

[5] Kawai, T., Nakashima, Y., Kokubo, Y., and Ohta, I., "Dual-band Wilkinson power dividers using a series RLC circuit," IEICE Trans. Electron., Vol. E91-C, No. 11, 1793-1797, 2008.

[6] Wu, Y., Liu, Y., and Li, S., “A new dual-frequency Wilkinson power divider," Journal of Electromagnetic Waves and Applications, Vol. 23, No. 4, 483-492, 2009.

[7] Fang, S., Guo, H., Liu, X. and Mao, L., "A miniaturized dual-frequency Wilkinson power divider using planar artificial transmission lines," Proc. of 2010 Int. Conf. on Microwave and Millimeter Wave Technology, 1588-1590, Chengdu, China, 2010.

[8] Tseng, C. H., Mou, C. H., Lin, C. C., and Chao, C. H., "Design of Microwave Dual-Band Rat-Race Couplers in Printed-Circuit Board and GIPD Technologies", IEEE Trans. Compon. Packag. Manuf. Tech., Vol. 6, No. 2, 262 - 271, Feb. 2016.

[9] Wu, G., Yang, L., Zhou, Y., and Xu, Q., "Wilkinson power divider for dual-band applications," IET Electron. Lett., Vol. 50, No. 14, 1003-1005, Jul. 2014.

[10] Li, X., Yang, Y.-J., Yang, L., Gong, S.-X., Tao, X., Gao, Y., Ma, K., and Liu, X.-L., "A novel design of dual-band unequal Wilkinson power divider," Progress In Electromagnetics Research PIER C, Vol. 12, 93-100, 2010.

[11] Gao, B., and Y. Liu, "Novel design of dual-band unequal Wilkinson power divider with wide band-ratios and simple layout," Proc. of 10th Int. Symp. on Antennas, Propagation \& EM Theory, 121-123, Xian, China, 2012.

[12] Kholodnyak, D., Kapitanova, P., Vendik, I., Humbla, S., Perrone, R., Müller, J., and Hein, M.A., "Novel Wilkinsontype power dividers based on metamaterial transmission lines," Proc. of 38th European Microwave Conf., 341-344, Amsterdam, Netherlands, 2008.

[13] Kholodnyak, D., Vorobev, E., Turgaliev, V., and Khalilova, E., "Design of a dual-band Wilkinson power divider using metamaterial transmission lines", Proc. of 2015 SBMO/IEEE MTT-S Int. Microwave and Optoelectronics Conf., 1-4, Porto de Galinhas - PE, Brazil, 2015.

[14] Chongcheawchamnan, M., Patisang, S., Krairiksh, M., and Robertson, I.D., "Tri-band Wilkinson power divider using a three-section transmissionline transformer," IEEE Microw. Wireless Compon. Lett., Vol. 16, no. 8, 452-454, Aug. 2006.

[15] Papanastasiou, A.C., Georghiou, G.E., and Eleftheriades, G.V., "A quadband Wilkinson power divider using generalized NRI transmission lines," IEEE Microw. Wireless Compon. Lett., Vol. 18, no. 8, 521-523, Aug. 2008. 